\title{
Proteomics for exotic organisms
}

A creative application of protein labeling can probe uncharacterized genomes.

Newts, with their ability to regrow amputated limbs and even internal organs, are an intriguing model for regeneration. But with a complex, unsequenced genome approximately ten times larger than the human genome, newts have seemed impenetrable to high-throughput proteomics studies. Now, researchers led by Thomas Braun at the Max Planck Institute in Bad Nauheim identify nearly 3,000 proteins in regenerating newt tails by adapting a labeling technique usually used to compare proteomes of cells grown under different treatment conditions.

Proteomes can be analyzed by digesting the proteins into peptides, identifying the pieces by mass spectrometry and then using database searches to match the peptides to proteins. This works well if many of an organism's gene and protein sequences are available, but attempts to match proteins across species tend to produce too many false identifications.

Mario Looso, a bioinformatician in Braun's laboratory, reasoned that stable-isotope labeling with amino acids in cell culture (SILAC) could be used to double-check such identifications. SILAC involves feeding cells or organisms nutrients containing a 'heavy' version of lysine to determine protein turnover or compare protein expression under different conditions. Braun, Looso and their colleagues used cells from unlabeled and labeled newts to hunt for matches to protein sequences contained in a newt expressed sequence tag library created in Braun's lab as well as for matches in databases containing sequences from zebrafish, frog, salamander, human and mouse. When the researchers found both 'heavy' and 'light' versions of the same peptides in their samples, they could be confident that their identifications were real. "Without that trick you would never know whether you are trying to find homologs to some junk material detected by the mass spectrometer," says Braun.

The researchers were able to compare protein expression in regenerating newt tails to undamaged tails. They are now using the method to study protein modifications and the regulation of protein turnover. For this, the ability to analyze material from growing organisms is incredibly important. "Most proteome techniques used cultured cells because of the difficulties to deal with complex 'real-life' in vivo systems," explains Braun. And the advantages should go beyond newts. "We hope that other researchers will use our technique to analyze the proteome of their favorite model organisms," he says.

Monya Baker

\section{RESEARCH PAPERS}

Looso, M. et al. Advanced identification of proteins in uncharacterized proteomes by pulsed in vivo SILAC. Mol. Cell. Proteomics advance online publication (5 February 2010). 